\section{Radiative Collisions of Neutrons and Protons}

IT has recently been shown by Lea ${ }^{1}$ that the passage of neutrons through paraffin wax and through liquid hydrogen gives rise to a gamma radiation of $1-6 \times 10^{6}$ e. volts energy, as well as recoil protons. As pointed out by Lea and Chadwick, the energy of these rays corresponds roughly to that which would be emitted in the radiative combination of a neutron and a proton to form a diplon. We have therefore calculated the probability of such a radiative collision on the assumption that the neutron behaves as a funda. mental charge-free particle throughout the collision, so that the radiation arises only from the acceleration of the proton by the field of force of the neutron. A dipole moment may then be associated with the system and the calculation carried out in the usual manner ${ }^{2}$. The result is that, for the range of energies involved in the experiments, combination should not take place more frequently than once in every 1000 collisions (the effective radius for diplon formation is about $2 \times 10^{-14} \mathrm{~cm}$.). This is much smaller than the observed frequency of about 1 in 4 collisions. We have also calculated the probability of a proton radiating in the impact without binding taking place, and find it to be even smaller. These results do not depend appreciably on the form of interaction assumed between neutron and proton.

It is of interest to note that if we assume that the neutron is a complex particle consisting of a proton and an electron, and that there are exchange forces between the neutron and proton of the type suggested by Heisenberg ${ }^{3}$, then we obtain a much smaller probability of combination (of the order of one effective collision in $10^{6}$ ), for with this model the dipole moment of the neutron-proton system vanishes ${ }^{4}$ In view of the discrepancy between theory and observation, and also in view of the different efficiencies to be expected for the process according as the neutron is or is not a fundamental particle, it is clearly important to obtain additional information about the nature of the neutron-proton collision.

H. S. W. Massey. C. B. O. MOHR.

Cavendish Laboratory, Cambridge. Jan. 18.

1 Nature, 133, 24, Jan. 6, 1934

"Mott and Massey, "The Theory of Atomic Collisions", p. 229

Z Z. Phys., 77, 1; 1932.

- Mott and Taylor, Proc. Roy. Soc., A, 138, 665 ; 1932.

\section{Remarkable Optical Properties of the Alkali Metals}

IN a recent communication, Zener ${ }^{1}$ has given an interesting interpretation of the peculiar optical properties of the alkali metals discovered by Wood ${ }^{2}$, differing from that which I previously suggested in these columns ${ }^{3}$. A closer consideration of the problem has led me to the conviction that the viewpoint of Zener, although not fully accounting for all the observed facts in its original form, can serve as a suitable basis for the discussion of the phenomena in question if modified in the following way :

Zener starts from the assumption that the con. duction electrons of the alkali metals are practically free. In the absence of temperature agitation of the lattice, they will then have only the one sharp resonance frequency, $\nu=0$, and no resonance frequencies corresponding to quantum jumps from the occupied to the unoccupied stationary states. As stated by Zener, the dielectric constant of the metal at frequency $\nu$ is in this case given by

$$
\varepsilon=1-\frac{N e^{2}}{\pi m v^{2}},
$$

where $N$ is the number of conduction electrons per unit volume, $e$ the electronic charge and $m$ the electronic mass. The electrical conductivity $\sigma$, giving the current in phase with the electric vector of the radiation, on the other hand, is zero for all frequencies except for $\nu=0$, where it becomes infinite. The metal will hence be totally reflecting, even at perpendicular incidence, for all frequencies for which $\varepsilon<0$, that is, below the frequency $\nu_{0}$ given by

$$
v_{0}^{2}=\frac{N e^{2}}{\pi m}
$$

Zener tries to account for the results of Wood, according to whom the transparency of thin films of the alkali metals, in going towards shorter wave. lengths, becomes greater by a factor of the order 100,000 within a rather narrow frequency interval in the ultra-violet, by means of this phenomenon of total reflection, finding from (1) values of $v_{0}$ which agree rather well with Wood's experimental data. The difficulty with this interpretation is that actually the alkali metals are by no means totally reflecting in the visible region, the coefficient of reflection in the case of potassium 4, for which the high transparency begins at about $3000 \mathrm{~A}$., decreasing from about 90 per cent at $5000 \mathrm{~A}$. to about 10 per cent at $2500 \mathrm{~A}$. Wood's results must therefore with certainty be ascribed to a change in the coefficient of extinction rather than to a change in the reflecting power.

Now such a change in the extinction coefficient can be obtained on the same fundamental assumptions as introduced by Zener, if it be remembered that the resonance frequency $\nu=0$ is not infinitely sharp but suffers a broadening due to the impacts of the conduction electrons with the metallic lattice, which the temperature agitation of the latter brings about. Specialising formulæ which I have given elsewheres for the case of free electrons, the half breadth $\delta$ of the resonance line $\nu=0$ is found to be

$$
\delta=\frac{N e^{2}}{2 \pi m \sigma_{0}},
$$

where $\sigma_{0}$ is the electrical conductivity for constant fields, while $\sigma$ and $\varepsilon$ are given by

$$
\sigma=\frac{\sigma_{0} \delta^{2}}{\nu^{2}+\delta^{2}}, \quad \varepsilon=1-\frac{2 \sigma_{0} \delta}{\nu^{2}+\delta^{2}} .
$$

The index of refraction $n$ and the coefficient of extinction $x$ are found from $\sigma$ and $\varepsilon$ by means of the relations ${ }^{8}$

$$
\begin{aligned}
& n^{2}=\frac{1}{2}\left(\sqrt{\varepsilon^{2}+4 \sigma^{2} / \nu^{2}}+\varepsilon\right), \\
& x^{2}=\frac{1}{2}\left(\sqrt{\varepsilon^{2}+4 \sigma^{2} / \nu^{2}}-\varepsilon\right) .
\end{aligned}
$$

In the table at the end of this letter I have computed the values of $\sigma, \varepsilon, n$ and $x$ for potassium in the interesting region of wave-lengths, using $\sigma_{0}=1.35 \times 10^{17}$, which leads to $\delta=4 \cdot 11 \times 10^{12}$ according to (2). As may readily be seen, there exists a critical frequency below which $n$ is abnormally small, while above it $x$ practically vanishes; the critical frequency with great approximation is equal to $v_{0}$ as given by (1). 